\title{
Rapid-stretch injury to peripheral nerves: comparison of injury models
}

\author{
Stewart Yeoh, PhD, Wesley S. Warner, BA, llyas Eli, MD, and Mark A. Mahan, MD \\ Department of Neurosurgery, Clinical Neurosciences Center, University of Utah, Salt Lake City, Utah
}

OBJECTIVE Traditional animal models of nerve injury use controlled crush or transection injuries to investigate nerve regeneration; however, a more common and challenging clinical problem involves closed traction nerve injuries. The authors have produced a precise traction injury model and sought to examine how the pathophysiology of stretch injuries compares with that of crush and transection injuries.

METHODS Ninety-five late-adolescent (8-week-old) male mice underwent 1 of 7 injury grades or a sham injury $(n>10$ per group): elastic stretch, inelastic stretch, stretch rupture, crush, primary coaptation, secondary coaptation, and critical gap. Animals underwent serial neurological assessment with sciatic function index, tapered beam, and von Frey monofilament testing for 48 days after injury, followed by trichrome and immunofluorescent nerve histology and muscle weight evaluation.

RESULTS The in-continuity injuries, crush and elastic stretch, demonstrated different recovery profiles, with more severe functional deficits after crush injury than after elastic stretch immediately following injury $(p<0.05)$. However, animals with either injury type returned to baseline performance in all neurological assessments, accompanied by minimal change in nerve histology. Inelastic stretch, a partial discontinuity injury, produced more severe neurological deficits, incomplete return of function, $47 \% \pm 9.1 \%$ (mean $\pm S D$ ) reduction of axon counts $(p<0.001)$, and partial neuroma formation within the nerve. Discontinuity injuries, including immediate and delayed nerve repair, stretch rupture, and critical gap, manifested severe, long-term neurological deficits and profound axonal loss, coupled with intraneural scar formation. Although repaired nerves demonstrated axon regeneration across the gap, rupture and critical gap injuries demonstrated negligible axon crossing, despite rupture injuries having healed into continuity.

CONCLUSIONS Stretch-injured nerves present unique pathology and functional deficits compared with traditional nerve injury models. Because of the profound neuroma formation, stretch injuries represent an opportunity to study the pathophysiology associated with clinical injury mechanisms. Further validation for comparison with human injuries will require evaluation in a large-animal model.

https://thejns.org/doi/abs/10.3171/2020.5.JNS193448

KEYWORDS peripheral nerve; stretch injury; neuroma; neuroma-in-continuity; nerve repair; nerve coaptation

$\mathrm{T}$ RAUMATIC nerve injury is a significant cause of pain and disability, with an estimated 18,000 new cases annually in the United States. ${ }^{1,2}$ Although peripheral nerves possess a remarkable facility for recovery and regrowth, the regenerative program may be impaired by the severity and mechanism of injury, resulting in limited or failed reinnervation in a majority of cases. Histopathological evaluation of injured nerves often reveals the formation of an intraneural fibrous scar, which has been associated with poor outcomes. ${ }^{3-5}$ Understanding the specific pathophysiology of failed regeneration is fundamental to advancing treatment options.

Nerve crush or transection injuries are common animal models used to elucidate the mechanisms of nerve regeneration. Crush injuries generally create a temporary dysfunction that recovers fully, ${ }^{6,7}$ whereas transection models show reinnervation ${ }^{3}$ and functional recovery. ${ }^{8}$ Although helpful in understanding successful reinnervation and neurological recovery, these models do not mimic the complexity and concomitant severity of clinical injuries. ${ }^{9}$ For example, one of the most frequent mechanisms of severe nerve injury results from rapid-stretch trauma sustained during falls or high-speed injuries, such as motor vehicle accidents. ${ }^{10,11}$ Because of the different injury mechanisms, animal models of crush or transection injuries do not develop the characteristic neuroma-in-continuity of clinical

ABBREVIATIONS DAPI = 4',6-diamidino-2-phenylindole; GLUT1 = glucose transporter-1; MBP = myelin basic protein; NF200 = neurofilament protein 200; SFI = sciatic function index. ACCOMPANYING EDITORIAL See pp 890-892. DOI: 10.3171/2020.6.JNS201799.

SUBMITTED February 19, 2020. ACCEPTED May 13, 2020.

INCLUDE WHEN CITING Published online November 6, 2020; DOI: 10.3171/2020.5.JNS193448. 
injuries, ${ }^{12,13}$ nor do they provide insight into the pathophysiology of closed stretch injuries. ${ }^{14}$

To address the disparity between clinical injury mechanism and basic science models, we have developed a reproducible, rapid-stretch nerve injury model for use in rodents that stratifies injury severity based on biomechanical characteristics. We have previously demonstrated that our model generates reproducible injury grades with histological features and functional outcomes similar to those of human nerve injuries. ${ }^{15}$ These animal models of stretch injuries exhibit long zones of fiber disruption that generate a disorganized matrix that shares many characteristics of clinical neuroma histology. ${ }^{12,16}$

To further validate this novel model, we compared rapid-stretch injury against established crush and transection protocols to highlight the histological and functional differences between injury mechanisms.

\section{Methods \\ Animals}

Ninety-five (n $>10$ per group) adolescent (8-weekold) male mice were used to simulate the primary clinical demographic for peripheral nerve injury. ${ }^{17}$ The transgenic B6.Cg-Tg(Thyl-YFP)HJrs/J; Jax mice (no. 003782; The Jackson Laboratory) that were used express a mosaic distribution of yellow fluorescent protein in the axons of motor and sensory fibers. Animals had ad libitum access to food and water while housed on a 12-hour light-dark cycle. Experiments were upheld to the standards of the University of Utah Institutional Animal Care and Use Committee.

\section{Nerve Injury Models}

Mice were anesthetized with $2 \%$ isoflurane and secured to a water-recirculating heating pad. The sciatic nerve was exposed from the knee to the sciatic notch with blunt dissection. One of 7 injury grades or sham injury, as described below, was applied to the nerve before closing the incision with nylon suture.

\section{Rapid-Stretch Injury}

Three grades of rapid-stretch injury were performed by using a weight drop on a cable and pulley/hook system, with severity graduated by maximum hook displacement: 1) elastic stretch injury (7.5-mm stretch excursion); 2) inelastic stretch injury (12.5 mm); and 3) stretch rupture $(>15 \mathrm{~mm})$. Nerve stretch injury was confirmed by calculation of maximum strain and persistent deformation, which were determined using high-speed (5000 fps) video of fiducial ink markers applied to the nerve before injury as previously described.$^{18}$ For rupture injury, nerve ends were reapproximated without suture or other coaptation.

\section{Crush Injury}

Crush injury was applied to the midsciatic nerve by using a titanium mini-aneurysm clip (Aesculap FT680T) with a jaw width of $1.0 \mathrm{~mm}$ and a crush force of $1.08 \mathrm{~N}$. Crush was applied for 30 seconds, after which the clip was removed and the incision closed.

\section{Primary Repair}

The midsciatic nerve was cut with sharp scissors and immediately coapted with two interrupted stitches through the epineurium with a 9-0 suture.

\section{Secondary Repair}

Transection was performed in the same manner as outlined for primary repair, except that after transection, proximal and distal nerve stumps were separated by 15 $\mathrm{mm}$ and lightly sutured to muscle on opposite sides of the incision. The wound was then closed, and the animals were returned to their home cages for 4 weeks to replicate delayed clinical repair. ${ }^{19,20}$ At 4 weeks the nerve was reexposed, and the proximal and distal stumps were carefully dissected from scar tissue. The nerve stumps were freshened to normal-appearing nerve and coapted with a 9-0 suture as discussed above. The 4-week delay in repair approximates $2-3$ years in humans. ${ }^{21}$

\section{Critical Gap}

To assess nerve discontinuity greater than critical gap distance, ${ }^{22,23}$ the nerves were exposed and a 15 -mm-long nerve segment was removed.

\section{Sham Injury}

Sham injury consisted of surgical exposure and placement of the nerve on the weight drop system hook for 30 seconds without rapid stretch.

\section{Behavioral Evaluation}

Animals were tested at a consistent time and acclimated to the testing room for 30 minutes before testing for all experimental time points. Functional performance was assessed by sciatic function index (SFI) for fine locomotor function according to the methods of Bain et al., ${ }^{24}$ in which mouse paw prints are analyzed for factors such as print length, step distance, and toe spread to determine a final SFI score. The tapered beam walking task was used to assess gross locomotor function, the plantar von Frey monofilament test for mechanical sensibility, and the Hargreaves method for temperature sensibility, as previously detailed..$^{18}$ Before nerve injury, animals underwent 2 days of training in which 2 trials of SFI and 3 trials with the tapered beam were performed. Baseline evaluation was obtained after 2 days of training (day -1 ), and all animals were trained to acceptable standards. A priori time points were determined for testing, as follows: after recovery from injury (day 0 ) and on days 1, 2, 6, 9, 15, 23, 30, and 48. For secondary repair, these time points were obtained after the delay in repair, except for baseline, which was obtained and recorded prior to initial surgery.

\section{Histological Assessment}

Animals were euthanized 48 days after injury. The injured sciatic nerve was extracted from anterior to the hamstring to posterior of the distal trifurcation. Proximal and distal segments (1-2 $\mathrm{mm}$ ) were reserved for axial sectioning for axon counts, and the zone of injury was sectioned longitudinally for evaluation of the injury architecture. Nerve histomorphometry, trichrome staining, and immu- 

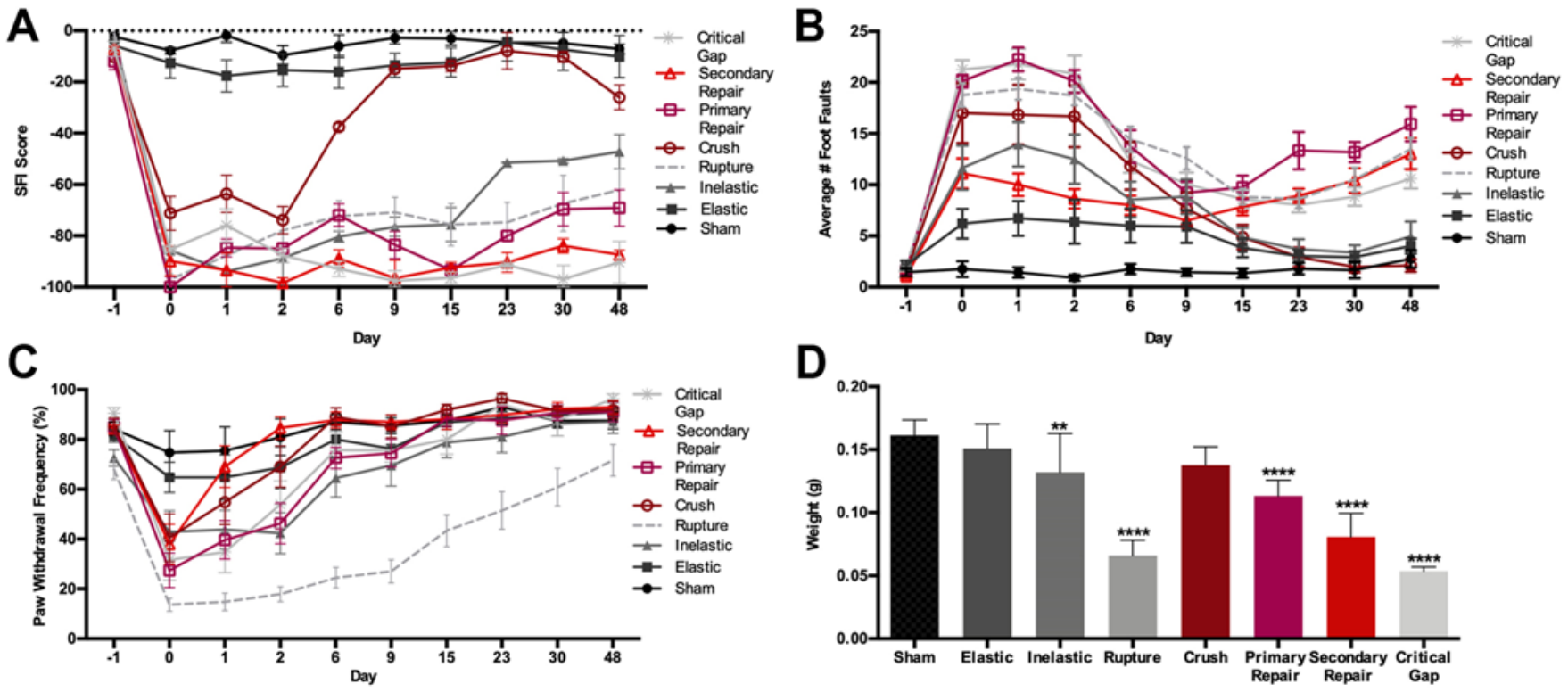

FIG. 1. Serial neurological assessment. A: SFI score. Animals with sham and elastic stretch injuries showed no significant difference in SFI scores across the testing period. Inelastic stretch generated significant $(p<0.01)$ acute impairment, with moderate recovery. Stretch rupture showed severe impairment $(p<0.001)$, with minimally recovering SFI over the testing period. In contrast, nerve crush generated only transient SFI deficit ( $p<0.05$ on days $0,1,2$, and 6$)$, recovering by day 9 . All transection models (primary repair, secondary repair, nerve gap) showed immediate and complete SFI impairment ( $<0.001)$. B: Tapered beam test. The performance of animals with sham injuries was consistent across testing days, and no difference from baseline was observed. Although an increase in foot faults was observed acutely after injury for elastic stretch, this behavior was not significant. Crush injury demonstrated significantly increased foot faults until day $6(p<0.01)$, and inelastic deficits persisted through day $9(p<$ 0.01 ), yet both injury grades recovered to baseline levels by day 15 . Primary repair, nerve gap, and rupture injury grades demonstrated markedly similar performances with significant deficits acutely after injury and progressive recovery until day $9(p<0.01$, $p<0.001, p<0.0001$, respectively). Although deficits remained statistically significant at day 15 , recovery proved to be transient and functional deficits worsened through the end of the testing paradigm (no repair, $p<0.05$; primary and stretch rupture, $p<$ 0.0001). Immediately after repair, secondary repair demonstrated a similar performance to nerve gap when compared at the same days after primary injury. Secondary repair followed with progressive deficits as other discontinuity injuries through the end of the experiment $(p<0.01)$. C: von Frey test. No difference in response was observed between sham and elastic injuries $(p=0.18)$. Crush injury demonstrated only sensation-related deficits the day of surgery $(p<0.05)$, whereas inelastic, primary, secondary, and nerve gap injuries demonstrated transient deficits $(p<0.05)$. A significant decrease in sensitivity was observed in stretch rupture $(p<0.01)$ until the final day of testing. D: Wet muscle weight. Crush and elastic injuries did not demonstrate a statistical decrease in gastrocnemius wet muscle weight in comparison with sham injuries. Inelastic injury demonstrated slight atrophy $(p<0.01)$, whereas the stretch rupture, primary, secondary, and nerve gap subgroups demonstrated statistically decreased muscle weight on harvest $(p<0.0001)$. Figure is available in color online only.

nofluorescent imaging were performed as previously described..$^{18}$ Specifically, Lille's trichrome imaging was used for evaluation of collagen deposition, cellularity, and comparison to clinical staining. Immunofluorescent antibodies were used to identify changes to structural components of the nerve after injury: perineurium (glucose transporter-1 [GLUT1], 1:200, Abcam); myelin (myelin basic protein [MBP], 1:500, Abcam); axons (neurofilament protein 200 [NF200], 1:500, Sigma); endoneurial extracellular matrix (laminin, 1:800, Sigma); and nuclei (4',6-diamidino-2-phenylindole [DAPI]). The gastrocnemius and tibialis anterior muscles of the injured and contralateral sides were harvested and weighed immediately for an indirect measurement of reinnervation.

\section{Statistical Evaluation}

GraphPad Prism 6 was used for statistical analysis. The Kruskal-Wallis test with Dunn's multiple comparison post hoc test was used to assess the tapered beam, von Frey, and Hargreaves performances; error bars are reported with standard error of the mean. SFI, wet muscle weight, and nerve fiber histomorphometry were assessed with one-way ANOVA (Tukey's post hoc test); error bars for these 3 values are reported with standard deviation. Significance was defined as $\mathrm{p}<0.05$.

\section{Results}

\section{Behavioral Evaluation}

Fine Locomotor Assessment (SFI)

Sham and elastic injuries demonstrated no statistically significant changes from baseline (Fig. 1A), whereas crush injuries exhibited a profound decrease in SFI immediately after injury $(-71 \pm 16[\mathrm{SD}], \mathrm{p}<0.001)$, with recovery to sham or baseline condition by day 9 . Severe rapid-stretch injury (inelastic stretch and stretch rupture) shared poor recovery with surgical discontinuity injuries (primary repair, secondary repair, and critical gap injuries), dem- 


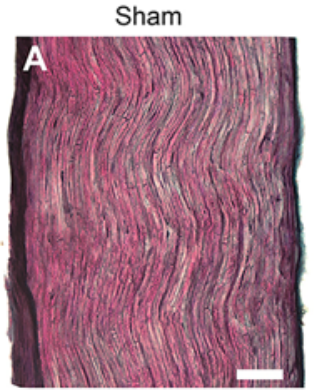

Crush

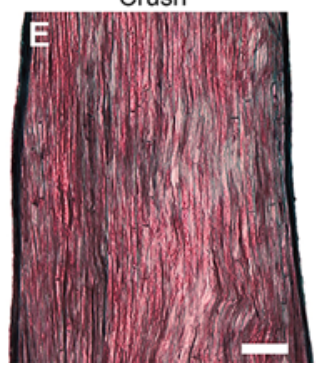

Elastic

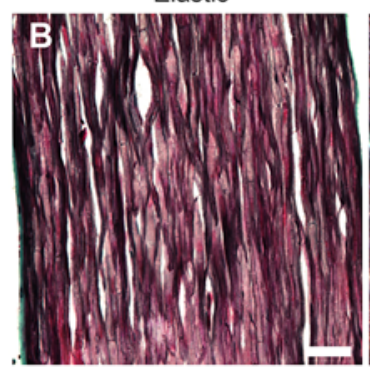

Primary Repair

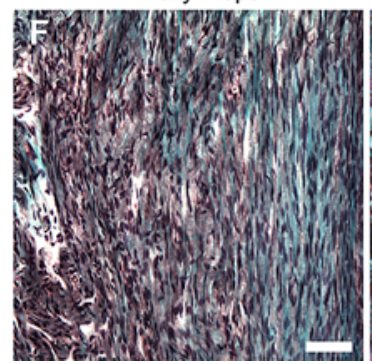

Inelastic

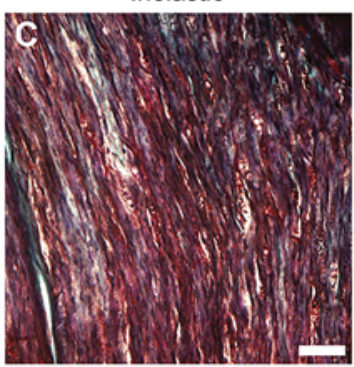

Secondary Repair

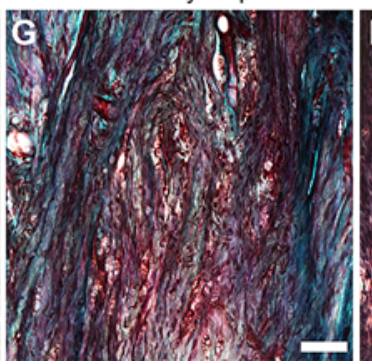

Rupture

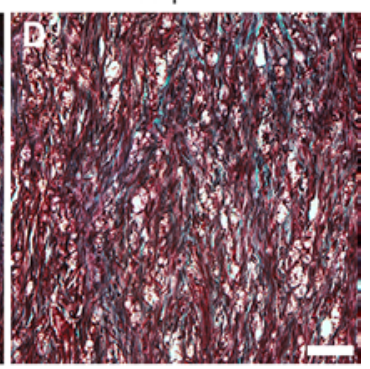

Critical Gap

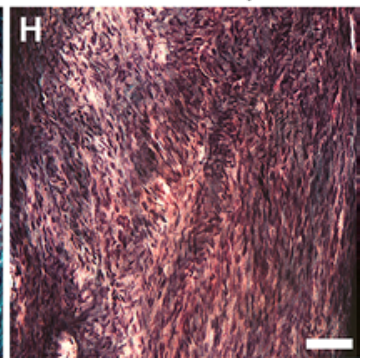

FIG. 2. Lillie's trichrome staining of longitudinally sectioned (15- $\mu \mathrm{m}$ slice thickness) sciatic nerve extracted 48 days after injury for assessment of fiber arrangement. Sham-injured nerves (A) demonstrated no change compared with normal control nerves (not shown). Elastic (B) and crush (E) injuries had intact fibers with no visible changes to the epineurium and perineurium. Inelastic injuries $(\mathbf{C})$, primary repair $(\mathbf{F})$, and secondary repair $(\mathbf{G})$ demonstrated partial fiber integrity; a number of fibers traverse the fringe of the injury and maintain uninterrupted connection. Other fibers are intensely snarled at the injury and suture site, suggestive of frustrated regeneration. Rupture (D) and critical gap $(\mathbf{H})$ demonstrated the most progressive pathology, with robust engorgement, collagen deposition, and fibrous entanglement characteristic of neuroma. Bar $=50 \mu \mathrm{m}$. Figure is available in color online only.

onstrating highly impaired function (inelastic $\mathrm{p}<0.01$; rupture, primary repair, secondary repair, nerve gap; $\mathrm{p}<$ 0.001) immediately after injury. Final outcomes ranged from moderate but highly variable impairment for animals in the inelastic injury group (SFI $-47 \pm 19, \mathrm{p}=0.07$ ) to severe impairment for all discontinuity models (rupture -71 \pm 23 , primary repair $-69 \pm 4$, secondary repair $-87 \pm 18$, nerve gap $-90 \pm 14$; all $p<0.001)$. There was no statistical difference between severe rapid-stretch injuries and surgical discontinuity injuries at the experimental endpoint.

\section{Gross Locomotor Assessment (Tapered Beam)}

All injuries demonstrated a transient increase in foot faults compared with sham injury (Fig. 1B). Elastic injury demonstrated only mild changes, whereas crush and inelastic injuries demonstrated significantly increased deficits until day $15(\mathrm{p}<0.01)$, but no statistical difference from sham injury at the experiment's endpoint. In contrast, discontinuity injuries (rupture, primary repair, secondary repair, and critical gap) demonstrated persistent deficits throughout the testing paradigm $(\mathrm{p}<0.01$, twoway ANOVA).

\section{Light Touch (von Frey)}

Sham and elastic injuries were not statistically different from one another, although both demonstrated a nonsignificant slight loss of sensation after surgery (Fig. 1C). Decreased sensation was observed in crush, inelastic, and secondary repair acutely after injury or repair; however, differences were not statistically significant $(p>0.05$ for all comparisons). Loss of sensation for primary repair and critical gap injuries was statistically significant on day 0 $(p<0.05)$, yet deficits were not significant on other testing days. Stretch rupture was the only injury to demonstrate significant and persistent sensory deficits through day 23 after injury $(\mathrm{p}<0.01)$.

\section{Temperature Sensitivity (Hargreaves)}

All animals retained sensitivity to heat stimulus after injury. Although instances of significant differences from sham injury were observed acutely after injury, no persistent or meaningful assumptions can be made with respect to injury grade.

\section{Histological Assessment \\ Wet Muscle Weight}

Rupture, primary and secondary repair, and critical gap injury were significantly atrophied in comparison with sham injury ( $<0.0001)$ (Fig. 1D). Inelastic injury also demonstrated a significant loss in wet muscle weight $(\mathrm{p}<0.01)$, whereas crush and elastic injuries were not statistically different compared with sham injury $(\mathrm{p}=0.07$ and $\mathrm{p}=0.81$, respectively).

\section{Trichrome Staining}

A positive association between fiber pathology and injury severity was observed in longitudinal sections of specimens (Fig. 2). Compact and oscillating fibers of sham-injured nerves recapitulated control nerve histology (not shown). Elastic and crush injuries demonstrated a similar pattern of loose and straightened endoneurial fibers, with intact external layers of perineurium and epi- 


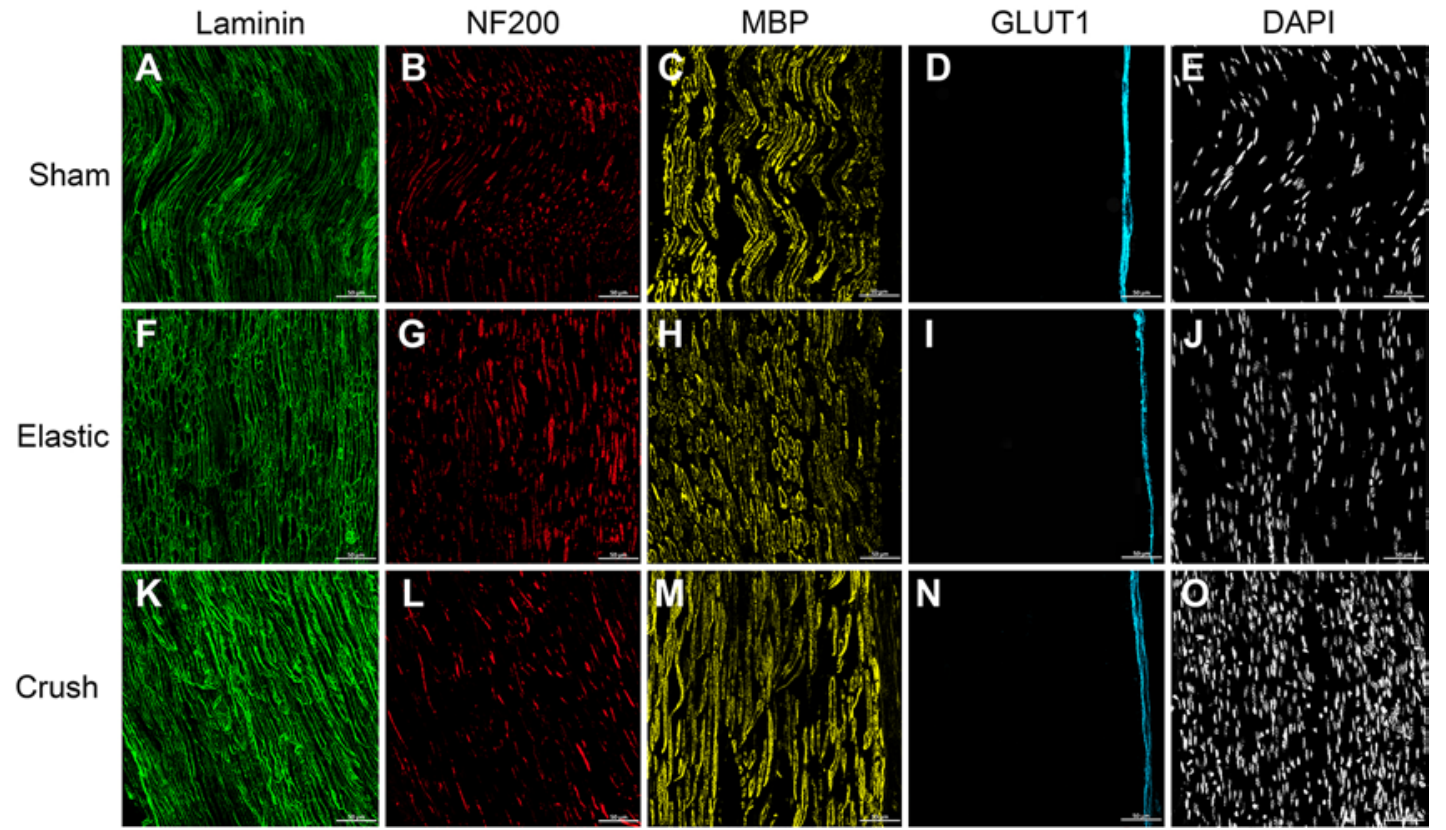

FIG. 3. Immunofluorescent longitudinal images (15- $\mu$ m slice thickness) of the area of maximal injury 48 days after injury for elastic and crush injuries. In comparison with the sham injury model (A-E), there were minimal architectural changes in elastic (F-J) and crush $(\mathrm{K}-\mathrm{O})$ injuries. Antilaminin depicted minimal change in endoneurial tube thickness and continuity in sham, elastic, and crush injuries $(A, F$, and $K)$. MBP $(C, H$, and $M)$ consistently encapsulated NF200 (B, G, and L) across injury grades; thus, MBP similarly attenuated as NF200 decreased with increasing injury severity. GLUT1 delineated the exterior perineurial layer of sham, elastic, and crush injuries, respectively $(D, I$, and $N)$. DAPI revealed hypercellularity in all injuries except elastic ones $(E, J$, and 0$)$. Original magnification $\times 200($ bar $=50 \mu \mathrm{m})$. Figure is available in color online only.

neurium. Inelastic, primary repair, and secondary repair injuries revealed extensive collagen deposition and disorganized fibers. For repair injuries, this phenomenon exists most prevalently around the suture site; however, for inelastic injuries, a dichotomy in pathology presentedwith one fascicle apparently uninjured, whereas the other fascicle manifested neuroma histology. Rupture and critical gap injuries were hallmarked by intense collagen deposition, dense and persistent hypercellularity, and fiber anarchy.

\section{Immunofluorescence Imaging}

Robust remodeling of nerve architecture increased with progressive injury severity (Figs. 3 and 4). Antilaminin depicted minimal change in endoneurial tube thickness and continuity in sham, elastic, and crush injuries. In contrast, inelastic injuries exhibited chaotic laminin architecture. In primary and secondary repair, laminin tubules proximal to the coaptation site were roughly the same diameter and resembled those of sham injury, with consistent linearity and continuity. However, around the site of coaptation, endoneurial tube integrity was compromised with a honeycomb-like matrix of laminin. Tubular morphology was not evident in rupture and critical gap injuries. NF200 identified continuous fibers in sham, elastic, and crush injuries. Axons were not linear and were more widely spaced in inelastic, primary, and secondary repair injuries, whereas they were seemingly nonexistent in rupture and critical gap injuries. Dense and tubular MBP enveloped NF200-positive axons in sham, elastic, and crush injuries. Attenuated NF200 was accompanied by a similar decrease of MBP at the coaptation site of primary and secondary repairs, as well as the intact portions of inelastic injuries. In contrast, dense MBP immunofluorescence and a distinct tube structure-similar to histological findings in sham injury-were observed both distal and proximal to coaptation. Rupture injuries demonstrated the most substantial reduction in MBP, whereas scattered MBP bodies were seen at the stump of the critical gap injury. GLUT1, a component of the perineurium and blood-nerve barrier, was restricted to the exterior of the nerve bundle in sham, elastic, and crush injuries. In contrast, GLUT1 infiltrated the endoneurium of severe stretch, primary, secondary, and critical gap injuries, crisply defining the endoneurial tubes throughout inelastic stretch-injured nerves. GLUT1 was sequestered primarily at the suture site in primary and secondary injuries and intensified around the suture material, which is a feature of a foreign body reaction (Fig. 5). ${ }^{25}$ Interestingly, although the ruptured nerves healed in continuity, there was no convincingly linear or tubular GLUT1 staining in the neuroma between the distal and proximal ends of ruptured nerves, as occurred after suture repair. DAPI staining showed minimal increase in cellularity in crush and elastic injuries in comparison with sham injuries, except at the site of the crush (Fig. 4). Primary, secondary, and critical gap injuries display hypercellularity similar to severe stretch injuries.

\section{Nerve Histomorphometry}

Sham-injured and elastic stretch-injured nerves demonstrated minimal change and did not differ significantly in number of axons (Fig. 6), average axon size, or myelin 


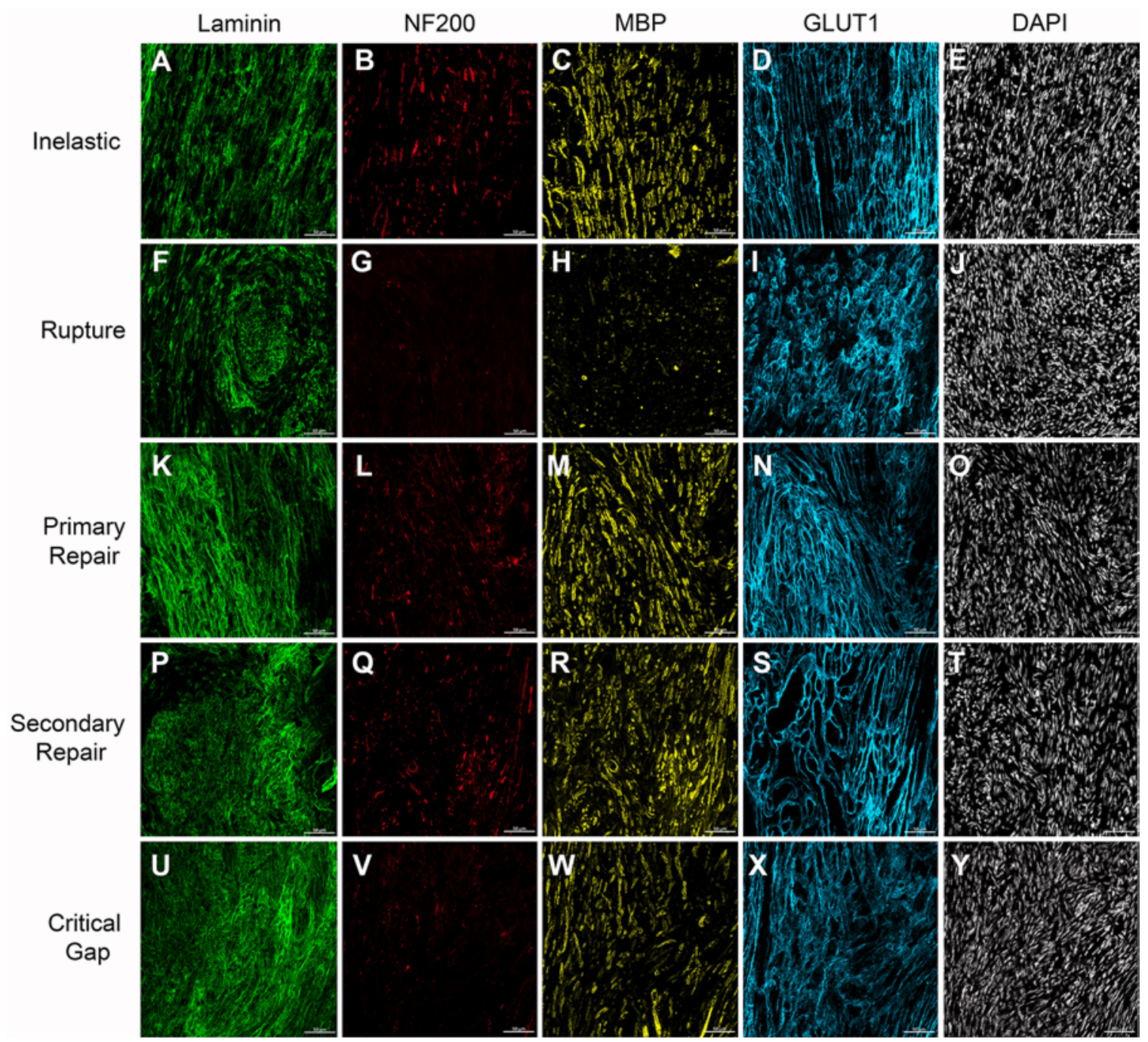

FIG. 4. Immunofluorescent longitudinal images (15- $\mu \mathrm{m}$ slice thickness) of the area of maximal injury 48 days after injury for severe stretch, repair, and critical gap. In comparison with the sham injury model (see Fig. $3 A-E$ ), severe stretch (inelastic [A-E] and rupture $[\mathrm{F}-\mathrm{J}])$, primary $(\mathrm{K}-\mathrm{O})$ and secondary $(\mathrm{P}-\mathrm{T})$ repair, and critical gap $(\mathrm{U}-\mathrm{Y})$ injuries demonstrated robust pathology. Laminin $(A, F, K, P$, and $U)$ and NF200 (B, G, L, Q, and V) immunofluorescence revealed aberrant remodeling of endoneurial tubules and limited axonal regeneration of primary and secondary repair and critical gap injuries, mirroring severe stretch injury. MBP $(C, H$, M, R, and W) consistently encapsulated NF200 across injury grades; thus, MBP similarly attenuated as NF200 decreased with increasing injury severity. Both MBP and NF200 were depleted in rupture injury, whereas some MBP persisted in critical gap injury despite the dearth of NF200. Profound GLUT1 infiltration hallmarks severe stretch injuries (inelastic and rupture), transection injuries (primary and secondary repair), and critical gap (D, I, N, S, and X). Interestingly, this infiltration demarcates the architecture of endoneurial tubules in inelastic, primary, and secondary repair, whereas stretch rupture and critical gap are typified by diffuse staining. Finally, DAPI revealed hypercellularity in all injuries except elastic ones (E, J, 0, T, and Y). Original magnification $\times 200$ $(\mathrm{bar}=50 \mu \mathrm{m})$. Figure is available in color online only.

thickness (data not included). All injury models except elastic had significantly $(\mathrm{p}<0.01)$ fewer axons distal to the injury zone. Additionally, inelastic, rupture, and critical gap injuries showed fewer axons proximal to the injury $(\mathrm{p}<0.01)$. There appears to be a loosely proportional increase in fibrous stroma of the distal nerve, with complete fibrous replacement of the distal nerve as a consequence of critical gap and rupture injuries.

\section{Discussion}

We previously defined the biomechanical properties of nerve subjected to rapid-stretch injuries, which fit into three thresholds of severity: elastic stretch, inelastic stretch, and stretch rupture..$^{15}$ The biomechanical severities reflected consistent patterns of acute histological changes ${ }^{26}$ that also correlated with longer-term neurological recovery and neuroma formation. Specifically, stretch within the elastic limit resembles a neurapraxic injury, whereas inelastic injury demonstrates cardinal features of axonotmesis with partial recovery. Stretch rupture of a nerve appears to obliterate successful regeneration..$^{18}$ Thus, the recovery potential of pure stretch injury follows the biomechanical and tissue structural properties of the nerve. 

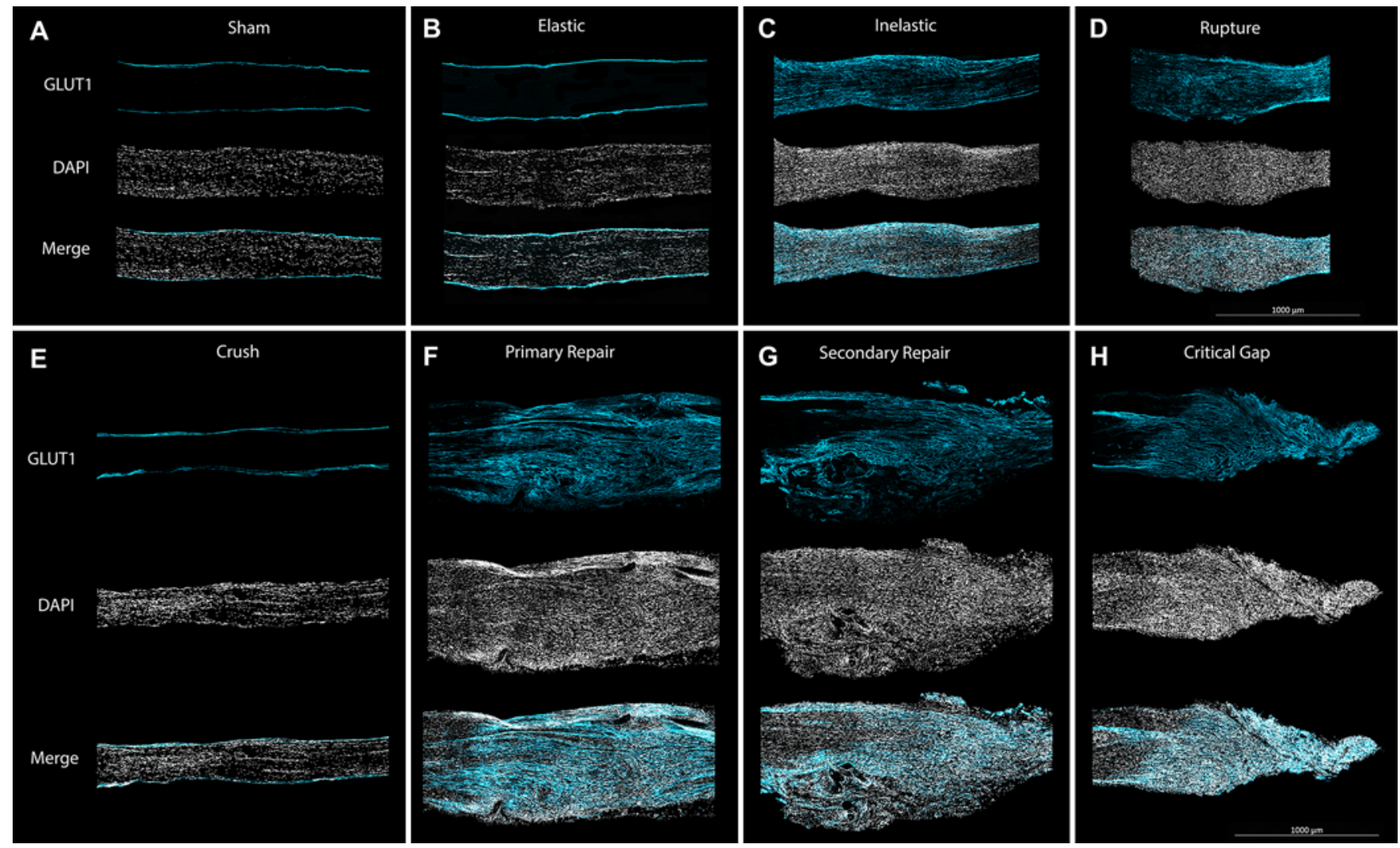

FIG. 5. Wide-field longitudinal immunofluorescent images (15- $\mathrm{mm}$ slice thickness) proximal and distal to the zone of maximal injury. Rich GLUT1 staining is consistent with the perineurium and distinctly outlined the nerve bundle in sham (A), elastic (B), and crush (E) injuries. In contrast, all other injuries demonstrated aberrant perineurial remodeling, with either GLUT1 outlining endoneurial tubes or chaotic GLUT1 infiltration. The endoneurial tubes are clearly defined in inelastic (C), primary repair (F), and secondary repair $(\mathbf{G})$ injuries. In nerve coaptations, this GLUT1 remodeling begins slightly proximal to the suture site, circumnavigates the suture, and then continues to infiltrate distally. In stretch rupture (D) and critical gap (H) injuries, we visualized chaotic GLUT1 infiltration at the neuroma site with progression to the distal aspect of the specimen. Original magnification $\times 100($ bar $=1000 \mu \mathrm{m})$. Figure is available in color online only.

In this study, we sought to examine how the graduated stretch injury severities compare with the traditional models used to study nerve regeneration: crush, transection, and critical gap. ${ }^{3,27}$ Our panel of behavioral and histological evaluations demonstrated a clear spectrum of injury, which can be summarized into three groups: nerve injury-in-continuity (elastic and crush), which demonstrated neurological recovery to baseline; partial discontinuity (inelastic), which demonstrated mixed recovery and histological outcomes; and discontinuity models (stretch rupture, primary and secondary repair, and critical gap), which shared similarly poor functional outcomes but divergent histopathological findings.

\section{Nerve-in-Continuity Injuries}

Crush injury is a common experimental nerve injury model because of its reproducibility and ease of surgical manipulation. ${ }^{28,29}$ Many studies demonstrate complete neurological recovery and little visible histopathological change. ${ }^{30-33}$ This provides an excellent comparison with elastic stretch injury, which similarly has shown scant neurological deficits and histopathological change, despite experiencing an average strain of nearly $50 \% .^{18}$
Crush is distinct from elastic stretch in the severity of deficits immediately after injury, with significantly worse performance in SFI, tapered beam, and von Frey testing. A focal crush injures axons and creates wallerian degeneration and regeneration, as observed by others. ${ }^{13,34,35}$ However, recovery after crush injury, as demonstrated by wet muscle weight, histology, and function returning to baseline, has been attributed to the preservation of endoneurial architecture, which facilitates successful guidance of regenerating axons to their prior distal targets. ${ }^{36}$ In support of this, we found that there were no histological changes to crushed nerves, except a small zone of persistently increased cellularity, which was suggestive of focal scar and potentially related to inflammation or the process of wallerian degeneration. ${ }^{37}$

In contrast, elastic stretch injuries demonstrated modest to insignificant behavioral alterations, presumably due to the distribution of stretching energy along the nervebased on continuum mechanics of stretch and our prior evaluation of histology immediately after stretch injuries. ${ }^{26}$ Axonal injury is presumably shielded by the internal redundancy of endoneurial undulations, the so-called bands of Fontana. ${ }^{38,39}$ Thus, the ephemeral functional deficits after elastic stretch injury recover too quickly to be at- 
Yeoh et al.
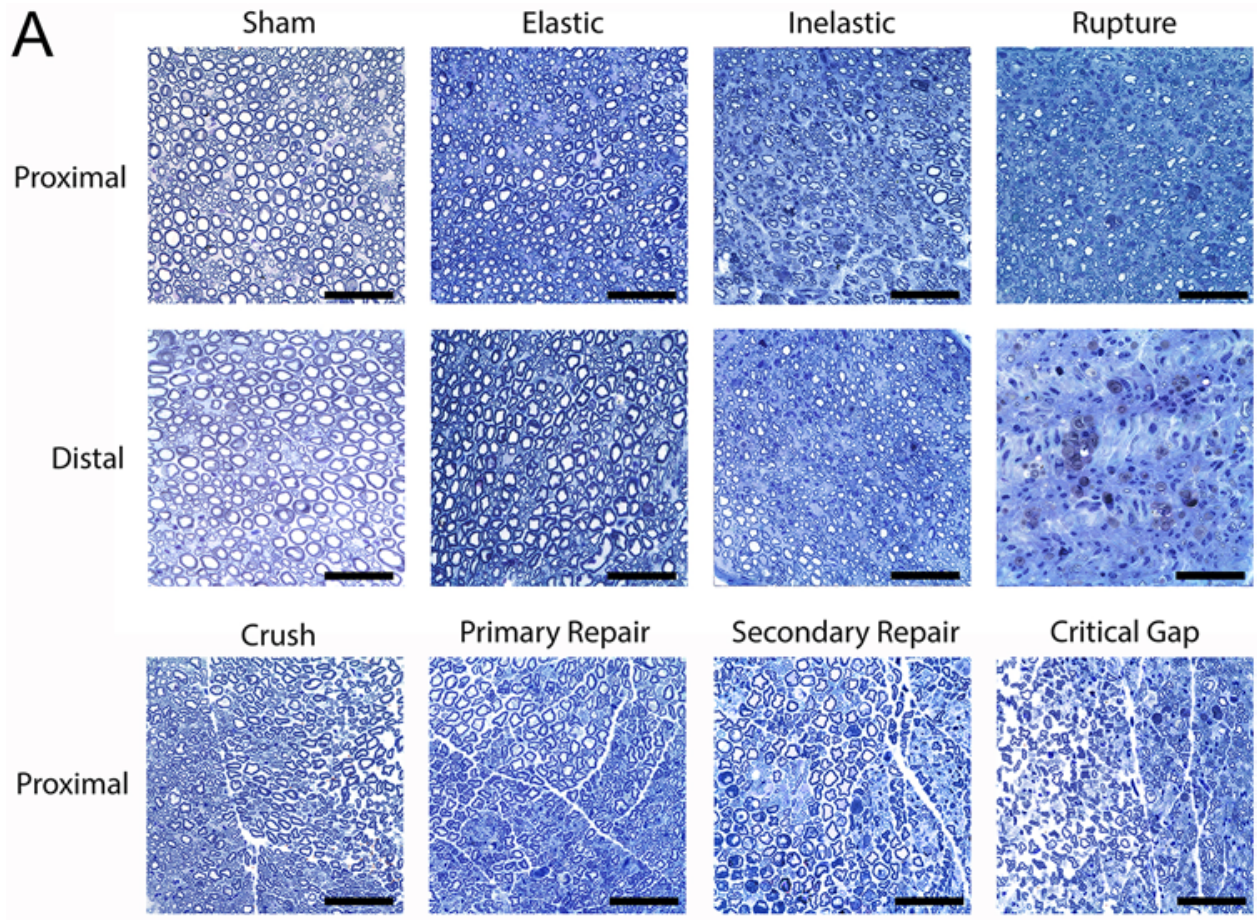

Critical Gap
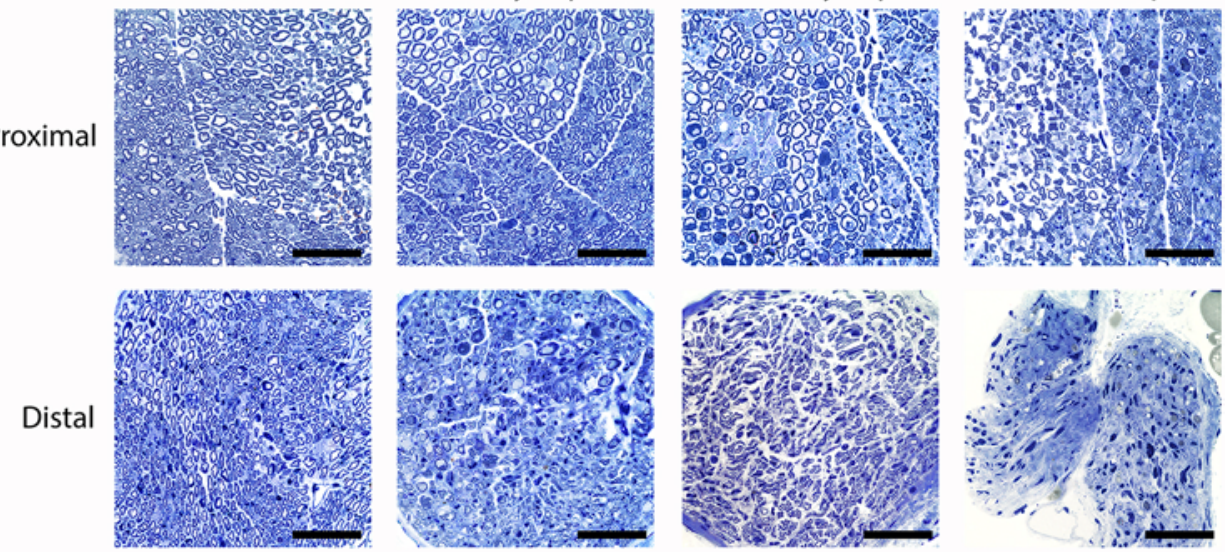

B
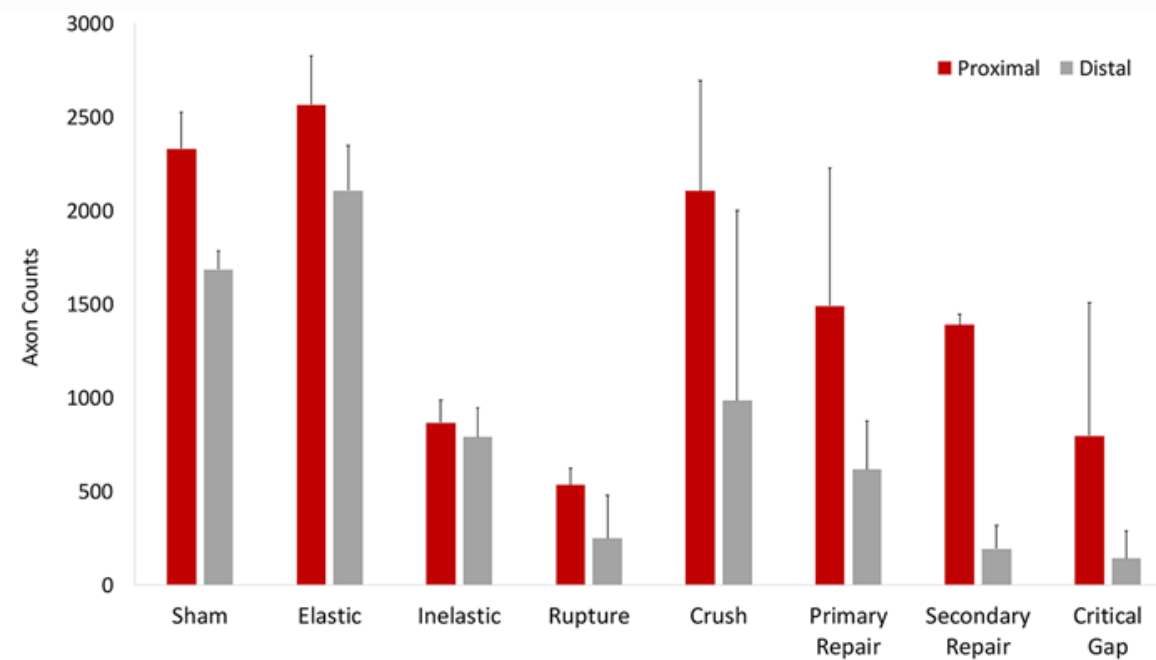

FIG. 6. A: Cross-sectional histomorphometry of sections far proximal and far distal to the zone of injury. Sham, elastic, and crush injuries retained large axons in proximal and distal sections. There were fewer large fibers in inelastic and primary and secondary repair injuries, and large fibers were effectively nonexistent in rupture or critical gap. In the distal segment, a greater degree of fibrous stroma accompanied the absence of large fibers. Osmium tetroxide staining, 1- $\mu$ m slice thickness, poststained in $1 \%$ toluidine blue before imaging. Original magnification $\times 400(\mathrm{bar}=50 \mu \mathrm{m})$. B: Histomorphometric analysis. Distal axon counts were significantly reduced $(p<0.01$ ) in all discontinuity (stretch rupture, primary and secondary repair, critical gap) and nerve crush models. Axon size decreased in repair, crush, and gap models, and myelin thickness decreased both proximally and distally in all models except elastic, with significant difference $(p<0.01)$ between proximal and distal in repair models (data not shown). Figure is available in color online only. 
tributed to substantial axon degeneration and regeneration, and are substantiated by minimal histological changes, similar to crush injury. The deficits after elastic stretch are more likely to be due to a conduction block from minute and reversible architectural changes, which may be further elucidated through electrophysiological investigation.

\section{Partial Discontinuity Injury}

Stretch beyond the limits of elasticity demonstrates internal fragmentation, ${ }^{26}$ as hallmarked by persistent elongation of the nerve. ${ }^{15}$ After longer survival, excised nerves display neuromatous areas as well as partially abnormal, linear axonal fibers, similar in appearance to elastic stretched nerves. ${ }^{18}$

This pattern of injury is unique with respect to all other injury mechanisms, in both neurological recovery and histology. Inelastic injury demonstrated more functional impairment and axonal loss than crush or elastic stretch, but less than discontinuity injuries. Muscle atrophy was similar to an in-continuity or successful repair of discontinuity injury. The inelastic injury was uniquely defined by deviation between functional results on the SFI and tapered beam test. Whereas all other injury grades either recovered (in-continuity injuries) or demonstrated persistent deficits (discontinuity injuries) for both functional tests, inelastic injury did not recover completely on the SFI test but did for the tapered beam. This dichotomized recovery suggests two considerations. First, the partial lesion of inelastic injury may provide for improved gross locomotor recovery. Second, SFI is a more sensitive test for differences in injury and recovery, despite the preservation of tactile sensibility after inelastic stretch.

The histological findings of inelastic injury shared features of both in-continuity and discontinuity injuries. First, one fascicle of the inelastic injury demonstrated continuity but apparent whorl-like neuroma in the other fascicle. The finding of intact but pathologically remodeled fascicles is a pattern we have previously observed in human neuroma-in-continuity specimens. ${ }^{12}$ Second, inelastic nerves demonstrated massive increases in cellularity (DAPI), perineurial cell (GLUT1) infiltration, and loss of axons (NF200) - as also seen in the discontinuity injuries. Myelination (MBP) is also reduced, characteristic of wallerian degeneration. This injury pattern may be most faithful to human neuroma-in-continuity lesions, in which nerve regeneration and consequent muscle and functional recovery are frequently partial and variable. ${ }^{9}$

\section{Discontinuity Injuries}

Primary (immediate) repair and secondary (delayed) repair represent models of clinical surgical repair and demonstrate histological evidence of axonal regeneration. In contrast, a critical gap injury is used as a negative control for regeneration studies. With a substantial part of the nerve removed, the likelihood of spontaneous regeneration is limited by the necessity of overcoming the critical gap. These extremes of discontinuity injuries were used for comparison with stretch-ruptured nerves.

Transection injuries demonstrated consistent and persistent deficits in function; however, motor testing did not distinguish between injuries with histological evidence of axonal regeneration (primary and secondary repair) and no axonal regeneration (critical gap and rupture). The persistent motor deficits after effective repair are consistent with findings from other groups ${ }^{29,31-33,40}$ and may indicate difficulty in reestablishing functional proprioception and motor control. Conversely, the apparent deficits may simply be the result of the animal's habituation or adaptation to the tests or the limitation of the tests as the mouse learns to walk with significant inversion or supination of the paw. Alternatively, poor performance could be explained by persistent pain as opposed to compromised function.

Plantar von Frey fiber testing revealed differences between the discontinuity injuries, yet created uncertainty in interpretation. Both primary and secondary repair demonstrated return of the withdrawal reflex to sham injury levels, which may reflect successful regeneration seen histologically. In particular, small-fiber regeneration appears to be more robust after transection. ${ }^{41}$ However, the recovery of withdrawal reflex in the critical gap injury suggests that alternative pathways may play a role, such as from the uninjured saphenous nerve. At the same time, the impoverished sensory response of stretch-ruptured nerves is evidence against this same conclusion. It may be that a combination of pain and deafferentation underlies these results; monofilament testing has been used to test both pain and sensation in rodent models. ${ }^{42}$ The experimental design could have benefited from additional sensation assessments to provide further detail, particularly concerning cautious interpretation of von Frey testing in nerve literature. ${ }^{43}$

Interestingly, although stretch-ruptured nerves healed in continuity, they showed no evidence of regeneration, which confirms that stretch rupture is a unique pathophysiology. Stretch rupture displayed impressive pathology characterized by complete absence of axons (NF200) and myelination (MBP), extensive perineurial (GLUT1) infiltration, riotous and occasionally swirled extracellular matrix (laminin), and a massive increase in cellularity (DAPI). This dysfunctional landscape shares histology with only the stump of the critical gap injury and the neuromatous portion of the inelastic injury. The haphazard matrix may represent a particular challenge for neurite outgrowth during regeneration, as suggested by others as the cause for poor recovery in humans. ${ }^{44}$

\section{Limitations}

We selected 48 days as the endpoint for functional and histological remodeling, based on a correlated duration of approximately 2 years in humans. ${ }^{21}$ Additional changes may manifest past the 48-day experimental window, as evidenced by the worsening tapered beam scores for all nerve discontinuity models. This abridged examination of recovery curves does not encompass the long-duration improvement and may miss additional changes, particularly in the nerve repair injury models. Eight-week-old animals were used to reflect the clinically predominant population of adolescents and young adults, because injury response and regenerative capacity appear to diminish with advancing age. ${ }^{45,46}$

Variation in injury severity may account for differences in outcome. For example, more severe crush or less suc- 
cessful surgical repair can create differential outcomes; however, our results are comparable to those of previously published rodent models. Stretch injuries were validated with postinjury video review; however, despite identical injury thresholds, the actual strain each nerve suffers is within a range and not identical to the others. Our initial characterization of stretch injury used cadaveric rat nerve tissue, ${ }^{15}$ and while some differences in elastic/inelastic strain thresholds and ultimate rupture limits were observed, rat and mice nerves were broadly similar in their response to stretch injury. ${ }^{18}$ Groups investigating nerve stretch injury should be aware that stretch magnitude, vector, and strain rate all will influence injury characteristics, and experiments should be designed accordingly. Electrophysiological testing may be helpful for evaluating the mechanisms underlying behavioral changes, and assessment of the dorsal root ganglion and spinal cord may reveal neuronal viability after the different mechanisms of nerve injuries. Both of these questions are under current investigation.

Last, direct application of these results to human clinical injuries is not appropriate. Human nerves have more connective tissue associated with the polyfascicular structure. Thus, stretch injuries in human nerves may have a different injury pattern. Further understanding of rapidstretch injuries in a large-animal model remains necessary for application to clinical injuries.

\section{Conclusions}

We compared the regenerative outcomes between biomechanically graded rapid-stretch nerve injuries and classic experimental models of nerve injury. Stretch-injured nerves differed substantially from existing models and demonstrate a distinct pathophysiology. For in-continuity injuries, crush injuries exhibited more substantial deficits, consistent with wallerian degeneration and regeneration, whereas elastic stretch produced a more subtle neurapraxia. When a nerve is stretched beyond its elastic limit, substantial fragmentation occurs. This partial discontinuity injury leads to diminished recovery, particularly fine-motor control, and histology consistent with partial neuroma-in-continuity. Full discontinuity injuries demonstrated a bifurcation in recovery. Transection with repair displayed axons crossing the gap and less significant muscle mass loss. In contrast, stretch rupture injury, although healed into continuity, demonstrated similar results to the negative control, which was critical gap injury. Stretchinjured nerves present a unique pathology and represent, in contrast to crush or transection, an opportunity to study the pathophysiology associated with clinical injury mechanisms. Further work is necessary to define the cellular proliferation and molecular cues inhibiting axonal regeneration.

\section{Acknowledgments}

We thank Kristin Kraus, MSc, for editorial assistance with this paper and Nancy Chandler for osmium sample preparation at the University of Utah Electron Microscopy Core Laboratory. This study was partially funded by the AANS/CNS Joint Section on Disorders of the Spine and Peripheral Nerves.

\section{References}

1. Karsy M, Watkins R, Jensen MR, et al. Trends and cost analysis of upper extremity nerve injury using the National (Nationwide) Inpatient Sample. World Neurosurg. 2019;123: e488-e500.

2. Foster CH, Karsy M, Jensen MR, et al. Trends and costanalysis of lower extremity nerve injury using the National Inpatient Sample. Neurosurgery. 2019;85(2):250-256.

3. Cattin AL, Burden JJ, Van Emmenis L, et al. Macrophageinduced blood vessels guide Schwann cell-mediated regeneration of peripheral nerves. Cell. 2015;162(5):1127-1139.

4. Parrinello S, Napoli I, Ribeiro S, et al. EphB signaling directs peripheral nerve regeneration through Sox2-dependent Schwann cell sorting. Cell. 2010;143(1):145-155.

5. Lin JC, Schwentker-Colizza A, Curtis CG, Clarke HM. Final results of grafting versus neurolysis in obstetrical brachial plexus palsy. Plast Reconstr Surg. 2009;123(3):939-948.

6. Brushart TM. Nerve Repair. Oxford University Press; 2011.

7. Dulin JN, Antunes-Martins A, Chandran V, et al. Transcriptomic approaches to neural repair. J Neurosci. 2015;35(41): 13860-13867.

8. Koka R, Hadlock TA. Quantification of functional recovery following rat sciatic nerve transection. Exp Neurol. 2001; 168(1):192-195.

9. Kim DH, Midha R, Murovic JA, et al, eds. Kline \& Hudson's Nerve Injuries: Operative Results for Major Nerve Injuries, Entrapments, and Tumors. 2nd ed. W.B. Saunders; 2007.

10. Noble J, Munro CA, Prasad VS, Midha R. Analysis of upper and lower extremity peripheral nerve injuries in a population of patients with multiple injuries. J Trauma. 1998;45(1): 116-122.

11. Birch R. Surgical Disorders of the Peripheral Nerves. Springer; 2011.

12. Mahan MA, Abou-Al-Shaar H, Karsy M, et al. Pathologic remodeling in human neuromas: insights from clinical specimens. Acta Neurochir (Wien). 2019;161(12):2453-2466.

13. Kline DG, Hayes GJ, Morse AS. A comparative study of response of species to peripheral-nerve injury. II. Crush and severance with primary suture. J Neurosurg. 1964;21: 980-988.

14. Kaplan HM, Mishra P, Kohn J. The overwhelming use of rat models in nerve regeneration research may compromise designs of nerve guidance conduits for humans. J Mater Sci Mater Med. 2015;26(8):226.

15. Mahan MA, Yeoh S, Monson K, Light A. Rapid stretch injury to peripheral nerves: biomechanical results. Neurosurgery. 2019;85(1):E137-E144.

16. van Vliet AC, Tannemaat MR, van Duinen SG, et al. Human neuroma-in-continuity contains focal deficits in myelination. J Neuropathol Exp Neurol. 2015;74(9):901-911.

17. Midha R. Epidemiology of brachial plexus injuries in a multitrauma population. Neurosurgery. 1997;40(6):1182-1189.

18. Mahan MA, Warner WS, Yeoh S, Light A. Rapid-stretch injury to peripheral nerves: implications from an animal model. J Neurosurg. Published online October 4, 2019. doi: 10.3171/2019.6.JNS19511

19. Woodhall B. Modern history of peripheral nerve surgery; World War II and the postwar study of peripheral nerve regeneration. J Am Med Assoc. 1949;139(9):564-566.

20. Kline DG. Timing for exploration of nerve lesions and evaluation of the neuroma-in-continuity. Clin Orthop Relat Res. 1982;(163):42-49.

21. Fox JG, Newcomer C, Smith A, et al, eds. The Mouse in Biomedical Research. 2nd ed. Elsevier; 2007.

22. Labroo P, Hilgart D, Davis B, et al. Drug-delivering nerve conduit improves regeneration in a critical-sized gap. Biotechnol Bioeng. 2019;116(1):143-154.

23. Madduri S, Gander B. Growth factor delivery systems and 
repair strategies for damaged peripheral nerves. J Control Release. 2012;161(2):274-282.

24. Bain JR, Mackinnon SE, Hunter DA. Functional evaluation of complete sciatic, peroneal, and posterior tibial nerve lesions in the rat. Plast Reconstr Surg. 1989;83(1):129-138.

25. Menovsky T, Beek JF. Carbon dioxide laser-assisted nerve repair: effect of solder and suture material on nerve regeneration in rat sciatic nerve. Microsurgery. 2003;23(2):109-116.

26. Warner W, Yeoh S, Light A, et al. Rapid-stretch injury to peripheral nerves: histologic results. Neurosurgery. 2020;86(3): 437-445.

27. Lewis GM, Kucenas S. Perineurial glia are essential for motor axon regrowth following nerve injury. J Neurosci. 2014; 34(38):12762-12777.

28. Geuna S. The sciatic nerve injury model in pre-clinical research. J Neurosci Methods. 2015;243:39-46.

29. Wolthers M, Moldovan M, Binderup T, et al. Comparative electrophysiological, functional, and histological studies of nerve lesions in rats. Microsurgery. 2005;25(6):508-519.

30. Gladman SJ, Huang W, Lim SN, et al. Improved outcome after peripheral nerve injury in mice with increased levels of endogenous $\omega-3$ polyunsaturated fatty acids. J Neurosci. 2012;32(2):563-571.

31. Inserra MM, Yao M, Murray R, Terris DJ. Peripheral nerve regeneration in interleukin 6-deficient mice. Arch Otolaryngol Head Neck Surg. 2000;126(9):1112-1116.

32. Yao M, Moir MS, Wang MZ, et al. Peripheral nerve regeneration in CNTF knockout mice. Laryngoscope. 1999;109(8): $1263-1268$.

33. Yao M, Inserra MM, Duh MJ, Terris DJ. A longitudinal, functional study of peripheral nerve recovery in the mouse. Laryngoscope. 1998;108(8 Pt 1):1141-1145.

34. Dailey AT, Avellino AM, Benthem L, et al. Complement depletion reduces macrophage infiltration and activation during Wallerian degeneration and axonal regeneration. J Neurosci. 1998;18(17):6713-6722.

35. Zochodne DW, Ho LT. Endoneurial microenvironment and acute nerve crush injury in the rat sciatic nerve. Brain Res. 1990;535(1):43-48.

36. Nguyen QT, Sanes JR, Lichtman JW. Pre-existing pathways promote precise projection patterns. Nat Neurosci. 2002;5(9): 861-867.

37. Moalem-Taylor G, Li M, Allbutt HN, et al. A preconditioning nerve lesion inhibits mechanical pain hypersensitivity following subsequent neuropathic injury. Mol Pain. 2011;7:1.

38. Alvey LM, Jones JFX, Tobin-O'Brien C, Pickering M. Bands of Fontana are caused exclusively by the sinusoidal path of axons in peripheral nerves and predict axon path; evidence from rodent nerves and physical models. J Anat. 2019;234(2): $165-178$.
39. Pourmand R, Ochs S, Jersild RA Jr. The relation of the beading of myelinated nerve fibers to the bands of Fontana. Neuroscience. 1994:61(2):373-380.

40. Kemp SW, Phua PD, Stanoulis KN, et al. Functional recovery following peripheral nerve injury in the transgenic Thyl-GFP rat. J Peripher Nerv Syst. 2013;18(3):220-231.

41. Lindenlaub T, Sommer C. Partial sciatic nerve transection as a model of neuropathic pain: a qualitative and quantitative neuropathological study. Pain. 2000;89(1):97-106.

42. Li Y, Dorsi MJ, Meyer RA, Belzberg AJ. Mechanical hyperalgesia after an L5 spinal nerve lesion in the rat is not dependent on input from injured nerve fibers. Pain. 2000;85(3): 493-502.

43. Deuis JR, Dvorakova LS, Vetter I. Methods used to evaluate pain behaviors in rodents. Front Mol Neurosci. 2017;10:284.

44. Tos P, Battiston B, Ciclamini D, et al. Primary repair of crush nerve injuries by means of biological tubulization with muscle-vein-combined grafts. Microsurgery. 2012;32(5):358-363.

45. Vaughan DW. Effects of advancing age on peripheral nerve regeneration. J Comp Neurol. 1992;323(2):219-237.

46. Verdú E, Ceballos D, Vilches JJ, Navarro X. Influence of aging on peripheral nerve function and regeneration. J Peripher Nerv Syst. 2000;5(4):191-208.

\section{Disclosures}

Dr. Mahan has served as a consultant for AxoGen and Joimax.

\section{Author Contributions}

Conception and design: Mahan, Yeoh, Warner. Acquisition of data: Yeoh, Warner, Eli. Analysis and interpretation of data: Mahan, Yeoh, Warner. Drafting the article: Mahan, Yeoh, Warner. Critically revising the article: Mahan, Yeoh, Warner. Reviewed submitted version of manuscript: Mahan, Yeoh, Warner. Approved the final version of the manuscript on behalf of all authors: Mahan. Figure production: Yeoh, Warner.

\section{Correspondence}

Mark A. Mahan: Clinical Neurosciences Center, The University of Utah, Salt Lake City, UT. mark.mahan@hsc.utah.edu. 